\title{
SUR UN THÉORÈME ÉTOILÉ DE GROSS
}

\author{
MAKOTO OHTSUKA
}

\section{Introduction}

Nous continuerons l'étude du premier théorème étoilé de Gross, faite dans le mémoire de l'auteur: 'Théorèmes étoilés de Gross et leurs applications, Ann. Inst. Fourier, 5 (1955), pp. 1-28; ce mémoire sera cité avec l'abréviation [A].

Dans la généralisation du théorème étoilé de Gross dans le théorème 1 de [A], on a considéré une représentation plus générale que représentation quasiconforme classique, d'un domaine plan de la forme de bande, noté $G$, dans une surface de Riemann. Dans le présent mémoire, on donnera d'abord une généralisation du théorème étoilé de Gross dans le cas où $G$ est transformé en un espace topologique qui contient des surfaces de Riemann homéomorphes à une partie de $G$, à l'aide de la notion généralisée de longueur extrémale, et après, on étendra le théorème 1 de [A] aux représentations continues, non nécessairement univalentes, localement pseudo-analytiques en dehors d'un certain ensemble exceptionnel au sens de Pfluger [6] et d'Ahlfors [1].

On s'occupera dans $\S 1$ du changement de variable dans une intégrale prise le long d'une courbe plane. Après cette préparation, pour une fonction $\pi(P)$ non négative sur un ensemble fini ou dénombrable de surfaces de Riemann, la longueur extrémale d'une famille de systèmes de courbes de poids $\pi(P) \geqslant 0$ sera définie, et il sera démontré que, si cet ensemble de surfaces de Riemann est transformé en un autre ensemble de surfaces de Riemann par un homéomorphisme de quotient de dilatation $q(P)$ généralisé, la longueur extrémale de poids $1 / q(P)$ d'une famille de systèmes de courbes n'est pas moins que la longueur extrémale de poids 1 de leurs images $(\$ 2)$. La positivité de la longueur extrémale d'une famille de systèmes de courbes qui séparent un continu dans $G$ d'un ensemble sur la frontière de $G$ sera montrée aussi dans $\S 2$. Ces résultats dans $\S 2$ nous permettent de généraliser le théorème étoilé de Gross pour des transformations de quotient de dilatation non nécessairement borné (§3). Ensuite, nous envisagerons la distance extrémale entre deux systèmes de courbes, et la longueur extrémale de la famille des courbes qui séparent ces deux systèmes, sur une surface de Riemann $(\$ 4)$. Les résultats seront appliqués à quelques questions de type de surfaces de Riemann $(\S 5)$.

Received July 12, 1955. 
§6 sera consacré à généraliser le théorème 1 de [A] aux représentations continues, localement pseudo-analytiques au sens de Pfluger-Ahlfors sauf en un certain ensemble exceptionnel. Enfin dans $\$ 7$, on améliorera certains des résultats obtenus dans [A], concernant la correspondance de frontières, en utilisant le théorème étoilé de Gross généralisé.

1. Nous commençons par justifier le changement de variable dans une intégrale prise le long d'une courbe plane. Soit $c=\{z(t) ; 0<t<1\}$ une courbe rectifiable située dans un domaine $D$ dans le plan $z=x+i y$, avec ses extrémités. A l'ordinaire, on définit la longueur $s(I)$ de l'image d'un intervalle $I:(0<) t_{1}<t<t_{2}(<1)$ par $\sup _{\delta} \sum_{i=1}^{n-1}\left|z\left(t^{i}\right)-z\left(t^{i+1}\right)\right|$ où $\delta$ est une division de $I: t^{1}$ $=t_{1}<t^{2}<\ldots<t^{n}=t_{2}$. A partir de cette fonction d"intervalle une mesure $s^{*}(B)$ sur la famille des ensembles boréliens dans $(0,1)$ est définie (voir [9], Chap. III); on l'appellera la mesure de longueur. Il est bien connu (voir [9], Chap. IV) que $s^{*}$ a la dérivée au sens général presque partout (p.p.), que $\lim _{\Delta t \rightarrow 0}|z(t+\Delta t)-z(t)| /|\Delta t|$ existe $\mathrm{p}$. p. et que tous les deux dérivées coüncident p. p. sur $(0,1)$. Étant donnée une fonction $\rho(z)$ réelle non négative sur $c$, l'intégrale inférieure $\int_{-c} \rho d s$ de $\rho(z)$ prise le long de $c$ est définie par le suprémum de l'intégrale de Lebesgue-Stieltjes $\int_{c} \underline{\rho} d s^{*}$, où $\underline{\rho}=\underline{\rho}(t)$ est une fonction borélienne non négative dans $(0,1)$, et non supérieure à $\rho(z(t))$.

Parmi les représentations équivalentes de $c$, il existe la représentation au moyen de la longueur d'arc: $z(s), 0<s<s_{0}$, où $s_{0}$ est. la longueur totale de $c$ (voir [8], p. 258). Pour cette représentation, $\lim _{\Delta s \rightarrow 0} \frac{|z(s+\Delta s)-z(s)|}{|\Delta s|}=1$ p.p. sur $0<s<s_{0}$, et $\int_{c} \rho d s$ est égale au suprémum de l'intégrale ordinaire de Lebesgue d'une fonction $\leqq \rho$, borélienne et non négative de $s$. De plus, on peut montrer que, $\rho(z)$ ㅆ 0 étant définie sur $c, \int_{0}^{1} \rho(z(t)) d s^{*}(t)$ est égale à $\int_{0}^{s_{0}} \rho(z(s)) d s$, n'importe quel paramètre $t$ étant choisi pour représenter $c$ à la manière equivalente.

Soit $f(z)$ un homéomorphisme de $D$ sur un domaine $G$ dans le plan $w=$ $u+i v$. On dira que $f(z)$ est absolument continu sur $c$, si $u(z(s))$ et $v(z(s))$ sont des fonctions absolument continues de $s$, et que $f(z)$ est totalement dérivable sur $c$ si $u(z)$ et $v(z)$ sont totalement dérivables en chaque point de $c$. Lorsque $f(z)$ est totalement dérivable en un point $z, D_{0} w=D_{\theta} w(z)=$ $\lim _{r \rightarrow 0}\left(f\left(z+r e^{i \theta}\right)-f(z)\right) / r e^{i \theta}$ existe pour tout $\theta, 0 \leqq \theta \leqq 2 \pi$, et égale $\left(u_{x} \cos \theta+\right.$ $\left.u_{y} \sin \theta\right)+i\left(v_{x} \cos \theta+v_{y} \sin \theta\right)$.

D'abord on prouve 
Lemme 1. Soit c une courbe rectifiable placée dans un domaine $D$ dans le plan $z$, avec ses deux extrémités, et soit $\rho(z)$ une fonction réelle non négative définie sur c. On transforme $D$ en un domaine dans le plan $w$ par un homéomorphisme $w=f(z)$ totalement dérivable et absolument continu sur c. Alors l'image $f(c)$ est rectifiable et on a

$$
\int_{0}^{s_{0}} \rho(z(s))\left|D_{\theta} w(z(s))\right| d s=\int_{f(c)} \rho\left(f^{-1}(w)\right) d s_{w},
$$

ò̀ $s_{w}$ est une mesure de longueur sur $f(c)$ et $D_{\theta} w(z(s))=D_{\theta} w(z)=\lim _{c \ni z^{\prime} \rightarrow z}\left(f\left(z^{\prime}\right)\right.$ $-f(z)) /\left(z^{\prime}-z\right)$, qui existe pour presque toute valeur de $s, 0<s<s_{0}$.

Il est immédiat que $f(c)$ est rectifiable lorsque $f(z)$ est absolument continue sur c. D'après le théorème de Radon-Nikodym, on a $s_{w}^{*}\left(B_{s}\right)=\int_{B_{s}} g(s) d s$ pour tout ensemble borélien $B_{s}$ dans $0<s<s_{0}$ avec une fonction borélienne $g(s)$ définie sur $0<s<s_{i}$. La dérivation des deux membres nous donne

$$
\begin{aligned}
g(s) & =\lim _{\Delta s \rightarrow 0} \frac{|f(z(s+\Delta s))-f(z(s))|}{|\Delta s|}=\lim _{\Delta z \rightarrow 0} \frac{|f(z+\Delta z)-f(z)|}{|\Delta z|} \cdot \lim _{\Delta s \rightarrow 0}\left|\frac{\Delta z}{\Delta s}\right| \\
& =\lim _{\Delta z \rightarrow 0} \frac{|f(z+\Delta z)-f(z)|}{|\Delta z|}=\left|D_{\theta} w(z(s))\right| \quad \text { p. p. dans } 0<s<s_{0} ;
\end{aligned}
$$

l'angle $\theta$ est égal à la pente de la tangente en point où celle-ci existe; c'est vrai p. p. sur $0<s<s_{0}$. Le changement de variable donne (1) (voir [9], p. 37).

2. Soit $\widetilde{\jmath}$ un ensemble fini ou dénombrable de surfaces de Riemann. On appellera un ensemble fini ou dénombrable de courbes sur $\tilde{F}$ un système de courbes. Soit $\{c\}$ une famille de systèmes de courbes sur $\widetilde{F}$. On définit une répartition sur $\widetilde{\gamma}$ par une quantité covariante non négative $\rho$ : pour chaque $z$ des paramètres locaux de $\widetilde{F}, \rho$ est une fonction $\rho(z)$ réelle non négative, et si $z$ et ${z^{\prime}}^{1)}$ sont deux paramètres locaux correspondant à un même domaine sur $\widetilde{F}$ et si $\rho(z)$ et $\rho^{\prime}\left(z^{\prime}\right)$ sont les représentations de $\rho$ en $z$ et $z^{\prime}$ respectivement, alors $\rho^{\prime}\left(z^{\prime}\right)=\rho(z)\left|d z / d z^{\prime}\right|$. Soit $c$ un élément de $\{c\}$. Il se compose d'un nombre fini ou dénombrable de courbes $\left\{c^{k}\right\}$. S'il existe une partie compacte de certaine $c^{k}$ représentée par un paramètre local en courbe non rectifiable, on pose $\int_{c} \rho d s=\infty$. Dans ce cas on dira que $c$ est localement non rectifiable. Sinon, d'après le lemme 1 , l'intégrale inférieure $\int_{c_{0}} \rho d s$ de $\rho$ prise le long d'une partie compacte $c_{0}$ quelconque de $c^{k}$ est bien définie; ici, on utilise le fait que, si $c_{0}$ est divisée en deux courbes $c_{1}+c_{2}$ ayant une extrémité commune, alors $\int_{c_{0}} \rho d s=\int_{c_{1}} \rho d s+\int_{c_{2}} \rho d s$. L'intégrale $\int_{c^{k}} \rho d s$ prise le long de $c^{k}$ sera définie par

1) Pour le moment, l'accent' ne signifie pas la dérivation. 
$\lim _{c_{0} \rightarrow c^{k}} \int_{c_{0}} \rho d s$, et $C_{\rho}(c)=\int_{c} \rho d s$ sera égale à $\sum_{k} \int_{c^{k}} \rho d s$.

Maintenant, $\pi(P)$ étant une fonction réelle non négative sur $\mathfrak{F}$, le module $M_{\pi}\{c\}$ de $\{c\}$ de poids $\pi$ est, par définition, $\underset{\rho}{\inf } \overline{\iint_{\mathscr{F}}} \pi \rho^{2} d x d y$ pour les répartitions $\rho$ sur $\widetilde{F}$ telles que $C_{\rho}(c) \geqslant 1$ pour tout $c \in\{c\}$; on dira que $\rho$ est une répartition en concurrence si cette condition est remplie; $\rho \in \infty$ est toujours une répartition en concurrence. Ici l'intégrale supérieure $\iint_{\mathscr{F}} \pi \rho^{2} d x d y^{2)}$ est égale à inf $\iint_{\mathscr{F}} \varphi d x d y$, où $\varphi$ est une fonction mesurable non inférieure à $\pi \rho^{2}$. La longueur extrémale $\lambda_{\pi}\{c\}$ de poids $\pi$ signifiera $1 / M_{\pi}\{c\}$. Lorsque, en particulier, $\pi \equiv 1$, on écrira simplement $M\{c\}$ et $\lambda\{c\}$.

Notre définition d'intégrales et donc les définitions de module et de longueur extrémale sont différentes de celles de Hersch [3], mais nos notions possèdent les propriétés analogues à celles des notions définies par Hersch [3].3) Par exemple:

a) Monotonie: si $\left\{c_{1}\right\} \subset\left\{c_{2}\right\}, \lambda_{\pi}\left\{c_{1}\right\}>\lambda_{\pi}\left\{c_{2}\right)$;

b) $M_{\pi}\left(\left\{c_{1}\right\} \cup\left\{c_{2}\right\}\right) \leqq M_{\pi}\left\{c_{1}\right\}+M_{\pi}\left\{c_{2}\right\}$;

c) si $c_{1} \subset \mathfrak{F}_{1} \subset \mathfrak{F}, c_{2} \subset \mathfrak{\mho}_{2} \subset \mathfrak{F}$ et $\mathfrak{F}_{1} \cap \mathfrak{F}_{2}=\phi$, alors $M_{\pi}\left(\left\{c_{1}\right\} \cup\left\{c_{2}\right\}\right)=M_{\pi}\left\{c_{1}\right\}+M_{\pi}\left\{c_{2}\right\}$

d) si $\left\{c_{1}\right\} \subset \mathfrak{F}_{1} \subset \mathfrak{F}_{,},\left\{c_{2}\right\} \subset \mathfrak{F}_{2} \subset \mathfrak{F}, \mathfrak{\mho}_{1} \cap \mathfrak{\mho}_{2}=\phi$ et chaque $c$ d'une famille $\{c\}$ contient au moins un $c_{1}$ et un $c_{2}$, alors $\lambda_{\pi}\{c\} \geqslant \lambda_{\pi}\left\{c_{1}\right\}+\lambda_{\pi}\left\{c_{2}\right\}$;

e) $\widetilde{F}_{1}$ étant un autre ensemble fini ou dénombrable de surfaces de Riemann, on transforme $\mathfrak{F}$ en $\widetilde{F}_{1}$ par un homéomorphisme $f(P)$, totalement dérivable et absolument continu sur tout élément de $\{c\}$ par rapport aux paramètres locaux. Soit $\left\{c_{1}\right\}$ la famille des images sur $\mathfrak{F}_{1}$ de $\{c\}$. En définissant le quotient de dilatation $q(P)$ en $P$, un point où $f(P)$ est totalement dérivable et possède le jacobien $J(z)=u_{x} v_{y}-u_{y} v_{x} \neq 0$ par rapport à un paramètre local $z=x+i y$ en $P$ et à un paramètre local $w=u+i v$ en $f(P)$, par $\max _{0 \leqq \theta \leqq 2 \pi}\left|D_{\theta} w(z)\right|^{2} /|J(z)|$, et par $\infty$ ailleurs sur $\mathfrak{\mho}$, on a $\lambda\left\{c_{1}\right\} \leqq \lambda_{1 / q}\{c\}$.

On se reporte à Hersch [3] pour les démonstrations de a)-d) et ici on démontre seulement e). Nous supposons que $\lambda\left\{c_{1}\right\}>0$. Soit $\rho_{1}$ une répartition en concurrence pour $c_{1}$; nous posons $\rho=\rho_{1} \max _{0 \leqq \theta \leqq 2 \pi}\left|D_{\theta} w\right|$ dans la partie de $\mathfrak{F}$ où $f(P)$ est totalement dérivable, et $=0$ ajlleurs sur $\mathfrak{F}$. Si $c$ est localement non rectifiable, certainement $\int_{c} \rho d s \geqq 1$. Soit donc $c$ localement rectifible. Alors

2) On pose $0 \times \infty=0$.

3) Voir ce mémoire pour la bibliographie sur la longueur extrémale et ses applications. Comme là, on peut définir le module d'un poids d'une famille numérique de courbes et en déduire ses propriétés comme ci-dessous. 
par le lemme $1,1 \leqq \int_{c_{1}} \rho_{1} d s_{1} \leqq \int_{c} \rho_{1} \max \left|D_{\theta} w\right| d s=\underline{\int}_{c} \rho d s$. En substituant $\rho$,

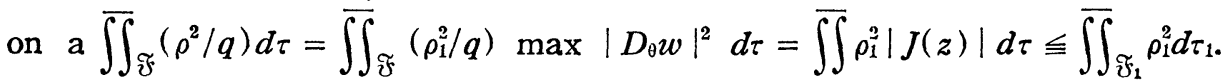
Donc $M_{1 / q}\{c\} \leqq M\left\{c_{1}\right\}$, d'où l'inégalité pour $\lambda$.

Le lemme suivant sera utile dans la démonstration du théorème 1 .

Lemme 2. Soit $G$ un domaine dans le plan $z=x+i y$ de la forme: $0<x$ $<1,0<y<h(x) \leqq \infty$, et soit $X$ un ensemble de mesure linéaire extérieure $\bar{m}(X)$ positive dans $0<x<1$ tel que, pour tout $x \in X, h(x)$ soit fini. Soit $K$ un continu dans $G$, et $\{\gamma\}$ l'ensemble des courbes joignant $K$ et l'ensemble $\Xi=$ $\{(x, h(x)) ; x \in X\}$ dans $G-K$ tel que chaque courbe aboutit à un point de $\Xi$ suivant un segment parallèle à l'axe des $y$. Alors la longueur extrémale de la famille des systèmes $\{c\}$ de courbes dont chaque $c$ se coupe toutes les courbes de $\{r\}$ est positive.

On choisit un sous-ensemble $X_{1} \subset X$ de $\bar{m}\left(X_{1}\right)>0$ tel que, pour tout $x \in X_{1}$, $h(x)$ soit inférieure à un nombre fini $Y>0$. Soit $R:(0<) x_{1}<x<x_{2}(<1)$, $0<y<y_{0}$ un rectangle dans $G$ tel que la partie $X_{2}$ de $X_{1}$ dans $\left(x_{1}, x_{2}\right)$ ait la mesure $\bar{m}\left(X_{2}\right)>0$ et que le continu $K$ donné soit placé hors $R$. On joint dans $G-K$ le point $\left(x_{1}, 0\right)$ à un point de $K$ par une courbe $c_{1}$ régulière placée en dehors de $R$, et le point $\left(x_{1}, y_{0}\right)$ à un autre point de $K$ par une courbe $c_{2}$ régulière, disjointe de $c_{1}$ et placée en dehors de $R$. On a ainsi un quadrilatère $Q$ de côtés suivants: $c_{1}+\left\{(x, y) ; x_{1}<x<x_{2}, y=0\right\} ;\left\{(x, y) ; x=x_{2}, 0<y<y_{0}\right\}$; $\left\{(x, y) ; x_{1}<x<x_{2}, y=y_{0}\right\}+c_{2}$; une partie de $K$. On transforme $Q$ conformément en un rectangle $Q_{0}: 0<\xi<a, 0<\eta<1$ par $\zeta=\zeta(z), \zeta=\xi+i \eta$, tel que le premier et le troisième des côtés de $Q$ correspondent aux côtés horizontals de $Q_{0}$. Pour le moment, on appellera l'image réciproque dans $Q$ d'un segment joignant deux côtés de $Q_{0}$ parallèle à l'axe des $\xi$ une ligne dans $Q$. On pose $\rho_{1}=1 / \bar{m}\left(X_{2}\right)$ dans la partie de $G$ appartenant au rectangle $0<x<1,0<y<Y$ et $=0$ ailleurs, et $\rho_{2}=\left|\frac{d \zeta}{d z}\right|$ dans $Q$ et $=0$ ailleurs, et on désigne par $\rho$ la superposition $\rho_{1}+\rho_{2}$. On montrera que $\rho$ est en concurrence pour la famille des systèmes $\{c\}$. Si un système $c$ ne se coupe pas un segment $\sigma:\{(x, y)$; $0<y<h(x), x=$ Cte $\left.\in X_{2}\right\}, c$ se coupe toutes les lignes dans $Q$; en effet, chaque ligne rencontre $\sigma$ et la réunion de la ligne et $\sigma$ contient une courbe de $\{r\} ; c$ doit se couper cette courbe et donc la ligne. Par suite, $\int_{c} \rho d s \geq \int_{c} \rho_{2} d s$ $=\int_{c}\left|\frac{d \zeta}{d z}\right| d s \geqslant 1$. Si $c$ rencontre tous les segments perpendiculaires aboutissant à $\Xi$, évidemment $\int_{c} \rho d s \geqq \int_{c} \rho_{1} d s \geqq 1$. Donc $\rho$ est en concurrence; $\iint_{G} \rho^{2} d x d y$ $\leqq 2 \iint_{G}\left(\rho_{1}^{2}+\rho_{2}^{2}\right) d x d y \leqq 2\left(\frac{Y}{\bar{m}\left(X_{2}\right)^{2}}+a\right)<\infty$ et le lemme est demontré. 
3. Soit $\widetilde{F}^{*}$ un espace topologique connexe, $\widetilde{\mho} \subset \mathfrak{F}^{*}$ un ensemble fini ou dénombrable de surfaces de Riemann, et $\left\{D_{n}\right\}$ une suite décroissante d'ensembles ouverts de frontières $\left\{c_{n}\right\}$ non vides, relatives à $\mathfrak{F}^{*}$, telles que $c_{1} \cap c_{n}=\phi(n \geqslant 2)$ et chaque $c_{n}$ se compose d'un nombre fini ou dénombrable de courbes régulières situées dans $\widetilde{F}$. Nous adjoignons à $\widetilde{F}^{*}$ un nouvel élément $\mathfrak{Z}$ et définissons sur $\mathfrak{F}^{*}+\{\Omega\}$ une topologie, en prenant $\left\{D_{n}+\{\Omega\}\right\}$ pour une base du filtre des voisinages de $\mathbb{L}$ et réservant les bases des filtres des voisinages des points de $\mathfrak{F}^{*}$. On dira que $\mathfrak{Q}$ est déterminé par $\left\{D_{n}\right\}$.

Le théorème suivant est une généralisation d'un théorème étoilé de Gross:

THÉORÈme 1. Soient $\widetilde{F}^{*}$, $\widetilde{J}$ et $\mathbb{Q}$ respectivement un espace topologique connexe, un ensemble fini ou dénombrable de surfaces de Riemann dans $\mathfrak{\mho}^{*}$, et l'élément déterminé par $\left\{D_{n}\right\}$ que nous venons de définir. Soit $G$ un domaine dans le plan $z=x+i y$ de la forme $0<x<1,0<y<h(x) \leqq \infty$, et soit $f(z)$ une application continue de $G$ dans $\mathfrak{F}^{*}$ telle que la restriction de l'application réciproque de $f(z)$ à $\widetilde{F}$ soit un homéomorphisme de $\widetilde{F}$. Soit $\left\{c^{n}\right\}$ une famille de systèmes de courbes dans $\tilde{\mho}$ séparant $c_{1}$ et $c_{n}$ sur $\mathfrak{F}^{* 1)}$ telle que $\lambda_{1 / q}\left\{c^{n}\right\} \rightarrow 0$ lorsque $n \rightarrow \infty$, où $q(P)$ est le quotient de dilatation du homéomorphisme $f^{-1}(P)$ de $\widetilde{\mho}$ dans $G$. On suppose que $f^{-1}(P)$ soit totalement dérivable et absolument continu sur tout $c^{n}$, par rapport aux parametres locaux. Si, pour chaque valeur $x$ d'un ensemble $X$ dans $0<x<1, h(x)$ est finie et quil existe une suite $y_{p} \rightarrow h(x)$ telle que $f\left(x+i y_{p}\right) \rightarrow \mathfrak{Q}$ lorsque $p \rightarrow \infty$ pour tout $x \in X$, alors la mesure linéaire $m(X)=0$.

En effet, considérons une composante connexe de l'image réciproque dans $G$ de $\mathfrak{F}^{*}-D_{1}$ et prenons dans elle un continu $K$. La propriété e) donne $\lambda_{1 / q}\left\{c^{n}\right\}$ $\geq \lambda\left\{c_{z}^{n}\right\}$, où $c_{z}^{n}$ est l'image réciproque de $c^{n}$. En désignant par $\{c\}$ la famille des systèmes de courbes définie dans le lemme 2 , on a $\lambda\left\{c_{z}^{n}\right\} \geqq \lambda\{c\}>0$ d'après a) et le lemme 2 , si l'on suppose $\bar{m}(X)>0$. C'est en contradiction avec l'hypothèse que $\lambda_{1 / q}\left\{c^{n}\right\} \rightarrow 0$ lorsque $n \rightarrow \infty$.

4. Dans le théorème 1 la condition que la longueur extrémale $\lambda_{1 / q}\left\{c^{n}\right\}$ tende vers 0 est essentielle. Le théorème 2 nous donnera une condition suffisante pour l'existence de $\left\{c^{n}\right\}$ telle que $\lambda_{1 / q}\left\{c^{n}\right\} \rightarrow 0$.

Lemme 3. Soit ₹̛ un ensemble fini ou dénombrable de surfaces de Riemann, et $u(P)$ une fonction harmonique sur $\mathfrak{F}$. Si $c(u)$ désigne la courbe de niveau: $u(P)=C t e u^{5)}$ si A est un certain ensemble de valeurs de u et si $\pi(P)$ est une fonction réelle non négative quelconque sur $\mathfrak{\mho}$, on a

4) On dit qu'un système de courbes sépare $c_{1}$ et $c_{n}$ sur $\mathfrak{F}^{*}$ lorsqu'il se coupe toutes les courbes joignant les points de $c_{1}$ et les points de $c_{n}$ sur $\mathfrak{F}^{*}$.

5) Elle consiste en un nombre fini ou dénombrable de courbes régulières. 


$$
\int_{A} \frac{d u}{\int_{c(u)} \frac{1}{\pi} d v} \leqq \frac{1}{\lambda_{\pi}\{c(u) ; u \in A\}},
$$

où $v(P)$ est la fonction harmonique conjuguée de $u(P)$ dans $\mathfrak{F}$.

En effet, soit $\rho$ une répartition en concurrence: $\int_{c(u)} \rho d s \geqq 1$ pour toute $c(u), u \in A$. Par l'inégalité de Schwarz, $1 \leqq\left(\int_{c(u)} \rho d v\right)^{2} \leqq \int_{c(u)} \pi \rho^{2} d v \cdot \underline{\int}_{c(u)} \frac{1}{\pi} d v$, et par suite $\int_{A} \frac{d u}{\int_{C(\mathcal{U})} \frac{1}{\pi} d v} \leqq \iint_{\int_{F}} \pi \rho^{2} d u d v$, d'où l'inégalité.

THÉORÈme 2. Soient $\mathfrak{F}^{*}$, $\mathfrak{F}$ et $\left\{D_{n}\right\}$ comme dans le théorème 1 ; nous posons $\Delta_{n}=D_{1}-D_{n}-c_{n}$. On suppose qu'on puisse trouver dans $\Delta_{n}$ une fonction $u_{n}(P)$ harmonique dans $\Delta_{n} \cap \tilde{\mho}$ telle que $\varlimsup_{c_{1}} u_{n}(P) \leqq 0$ et $\frac{\lim }{c_{n}} u_{n}(P) \geqq 1$ et que $\bar{\int}_{A_{n}} d u_{n} / \int_{c\left(u_{n}\right)} q d v_{n} \rightarrow \infty$, ò̀ $A_{n}$ est l'ensemble de valeurs de $u_{n}, 0<u_{n}<1$, tel que l'ensemble $\left\{P \in \mathfrak{F}^{*} ; u_{n}(P)=\right.$ Cte $\left.u_{n}\right\}$ soit situé dans $\Delta_{n} \cap \mathfrak{F}$ pour toute $u_{n} \in A_{n}$. Si on prend pour $\left\{c^{n}\right\}$ les courbes de niveau $\left\{c\left(u_{n}\right) ; u_{n} \in A_{n}\right\},\left\{c^{n}\right\}$ remplit les conditions de l'hypothèse du théorème 1.

En effet, sur toute courbe $\gamma$ joignant $c_{1}$ et $c_{n}$ dans $\Delta_{n}$ la fonction $u_{n}(P)$ varie continûment de 0 à 1 , et donc toute $c^{n}$ rencontre $\gamma$. De plus, on voit $\lambda_{1 / q}\left\{c^{n}\right\} \rightarrow 0$ par le lemme précédent.

Dans la suite on étudiera en détail le cas particulier où $\mathfrak{\mho}^{*}=\mathfrak{F}$, une surface de Riemann et $q(P) \equiv 1$. Soit $\Delta$ un ensemble ouvert dans $\widetilde{F}$, soient $c_{1}$ et $c_{2}$ deux systèmes disjoints de courbes régulières dans $\widetilde{\mho}$ qui forment la frontière relative de $\Delta^{6)}$ soit $\{c\}$ la famille des systèmes de courbes dans $\Delta$ homologues à $c_{1}$, à une partie de la frontière idéale de $\Delta$ près, ${ }^{7)}$ et soit $\{\gamma\}$ la famille des courbes joignant $c_{1}$ et $c_{2}$ dans $\Delta$. On démontrera qu'on peut trouver une fonction extrémale harmonique $u_{0}(P)$ qui donne le maximum à l'intégrale à gauche de $(2)$ et telle que la valeur de l'intégrale soit égale à $1 / \lambda\{c\}$ et aussi bien qu'à $\lambda\{\gamma\}$.

D'abord on suppose que $\Delta$ ne soit pas compact dans $\widetilde{F}$ et on fait une exhaustion de $\widetilde{F}$ par les domaines $\left\{B_{p}\right\}$ de frontières régulières compactes $\left\{\Gamma_{p}\right\}$. On pose $B_{p} \cap \Delta=\Delta_{p}, B_{p} \cap c_{1}=c_{p}^{1}, B_{p} \cap c_{2}=c_{p}^{2}$ et $\Gamma_{p} \cap \Delta=\gamma_{p}$. Soit $u_{p}(P)$ la fonction harmonique bornée sur $\Delta_{p}$, égale à 0 sur $c_{p}^{1}$ et à 1 sur $c_{p}^{2}$ et de dérivée normale $\frac{\partial u_{p}}{\partial \nu}=0$ sur $\gamma_{p}$. Par la formule de Green, l'intégrale $\int \frac{\partial u_{p}}{\partial \nu} d s$ est

6) Lorsque $\Delta$ est placé aux deux côtés d'un arc de $c_{1}$ ou $c_{2}$, on compte cet arc deux fois comme une partie de la frontière de $\Delta$.

7) Cela veut dire que $c$ et $c_{1}$ ensemble forment la frontière relative à $\mathfrak{F}$ d'un certain sous-ensemble ouvert de $\Delta$. 
constante sur toute courbe homologue à $c_{p}^{1}$, à une partie de $\gamma_{p}$ près, et égale à l'intégrale de Dirichlet $D_{\Delta_{p}}\left[u_{p}\right]$. Pour $q>p$ il s'en suit encore par la formule de Green que $0<D_{\Delta_{p}}\left[u_{q}-u_{p}\right]=D_{\Delta_{p}}\left[u_{q}\right]-D_{\Delta_{p}}\left[u_{p}\right]$. Alors $D_{\Delta_{p}}\left[u_{p}\right]$ est croissante. Si sa limite est finie, on peut conclure par le raisonnement habituel que $u_{p}(P)$ tend vers une fonction harmonique $u_{0}(P)$ localement uniformément sur $\Delta+c_{1}+c_{2}$, et que $\lim _{p \rightarrow \infty} D_{\Delta_{p}}\left[u_{p}\right]=D_{\Delta}\left[u_{0}\right]$. Alors $u_{0}(P)=0$ sur $c_{p}^{1}$ et $=1$ sur $c_{p}^{2}$. On note aussi que, parmi les fonctions $\{u(P)\}$ harmoniques dans $\Delta$ telles que $\varlimsup_{c_{1}} u(P) \leqq 0$ et $\frac{\lim }{c_{2}} u(P) \nRightarrow 1, u_{0}(P)$ est la seule fonction qui donne l'intégrale de Dirichlet minimale. Si $D_{\Delta_{v}}\left[u_{p}\right] \rightarrow \infty$, on choisit une suite partielle de $\left\{u_{p}(P)\right\}$ convergente vers une fonction harmonique $u_{0}(P)$ localement uniformément sur $\Delta+c_{1}+c_{2}$; c'est possible par le théorème de Harnack, car $u_{p}(P)$ sont uniformément bornées. Comme $D_{\Delta}\left[u_{0}\right] \geqq D_{\Delta_{p}}\left[u_{p}\right] \rightarrow \infty, D_{\Delta}\left[u_{0}\right]=\infty$.

Comme $c \cap \Delta_{p}$ est homologue à $c_{p}^{1}$ dans $\Delta_{p}$ pour $c \in\{c\}$, à une partie de $\gamma_{p}$ près, on a $\int_{c \cap \Delta_{p}} d v_{p}=D_{\Delta_{p}}\left[u_{p}\right]$, où $v_{p}(P)$ est la fonction conjuguée de $u_{p}(P)$. Si on pose $\rho=1 / D_{\Delta_{p}}\left[u_{p}\right]$ dans $\Delta_{p}$ par rapport au paramètre $u_{p}+i v_{p}$ et $=0$ ailleurs, on a $1 \leqq \int_{c} \rho d s$ et donc $1 / \lambda\{c\} \leqq \iint_{\Delta_{p}} \rho^{2} d u_{p} d v_{p}=1 / D_{\Delta_{p}}\left[u_{p}\right]$, d'où $\lambda\{c\} \geqslant \lim _{p \rightarrow \infty} D_{\Delta_{p}}\left[u_{p}\right]$ $=D_{\Delta}\left[u_{0}\right]$.

Soit $u(P)$ une fonction harmonique dans $\Delta$ telle que $\overline{\lim }_{c_{1}} u(P) \leqq 0$ et $\lim _{c_{2}} u(P)$ $\geqslant 1$, soit $v(P)$ la fonction conjuguée, et soit $c(u)$ la courbe de niveau: $u(P)=$ Cte $u, 0<u<1$, dans $\Delta$. Le lemme 3 montre que $\int_{0}^{1} d u / \int_{c(u)} d v \leqq 1 / \lambda\{c(u)\}$. D'autre part, comme toute $c(u) \in\{c\}$, on voit que $\lambda\{c(u)\} \geqslant \lambda\{c\}$. Donc, en vertu de l'inégalité $\lambda\{c\} \geqslant D_{\Delta}\left[u_{0}\right]$ obtenue ci-dessus, on en déduit

$$
\int_{0}^{1} \frac{d u}{\int_{c(u)} d v} \leqq \frac{1}{\lambda\{c\}} \leqq \frac{1}{D_{\Delta}\left[u_{0}\right]} .
$$

On va démontrer que $u_{0}(P)$ est la fonction extrémale qui donne à l'intégrale la valeur maximum, égale à $1 / \lambda\{c\}=1 / D_{\Delta}\left[u_{0}\right]$. Pour cela il est suffissant de savoir que $\int_{c\left(u_{0}\right)} d v_{0}=D_{\Delta}\left[u_{0}\right]$ pour presque toute valeur de $u_{0}$. Si $D_{\Delta}\left[u_{0}\right]=\infty$, l'intégrale à gauche de (3) est zéro et donc $\int_{c\left(u_{0}\right)} d v_{0}=\infty$ pour presque toute valeur de $u_{0}, 0<u_{0}<1$. Si $D_{\Delta}\left[u_{0}\right]<\infty$, on prend une partie compacte $c^{\prime}$ de $c\left(u_{0}\right)$ telle que $\int_{c\left(u_{0}\right)} d v_{0}<\int_{c l} d v_{0}+\varepsilon$ lorsque la valeur à gauche est finie. Comme $u_{p}(P)$ et ses dérivées partielles tendent localement uniformément vers $u_{0}(P)$ et vers ses dérivées partielles respectivement, $\int_{c^{\prime}} d v_{0}=\lim _{p \rightarrow \infty} \int_{c^{\prime}} d v_{p}=\lim _{p \rightarrow \infty} \int_{c_{\nu^{\prime}}} d v_{p} \leqq$ 
$\lim _{p \rightarrow \infty} \int_{u_{p}(P)=u_{0}} d v_{p}=\lim _{p \rightarrow \infty} D_{\Delta_{p}}\left[u_{p}\right]=D_{\Delta}\left[u_{0}\right]$, où $c_{p}^{\prime}$ est la partie de la courbe de niveau $u_{p}(P)=u_{0}$ dont tout point est joint à un point de $c^{\prime}$ par une courbe $v_{p}(P)$ $=$ Cte. Donc $\int_{c\left(u_{0}\right)} d v_{0} \leqq D_{\Delta}\left[u_{0}\right]$. On peut montrer par le même raisonnement que l'intégrale est toujours finie et donc toujours $\leqq D_{\Delta}\left[u_{0}\right]$. En considération de (3) vraie pour $u_{0}$ et $v_{0}$, on a $\int_{c\left(u_{0}\right)} d v_{0}=D_{\perp}\left[u_{0}\right]$ pour presque toute valeur de $u_{0}, 0<u_{0}<1$.

Ensuite on peut démontrer que la distance extrémale $\lambda\{\gamma\}=1 / D_{\Delta}\left[u_{0}\right]$. En effet, en considération de la répartition $\rho=1$ par rapport à $u_{0}+i v_{0}$, on déduit l'inégalité $\lambda\{\gamma\} \geqslant 1 / D_{\perp}\left[u_{0}\right]$. Si $\rho$ est une répartition en concurrence pour $\{\gamma\}$, représentée comme une fonction de $u_{p}+i v_{p}$ dans $\Delta_{p}$, et si

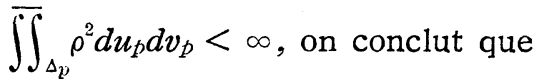

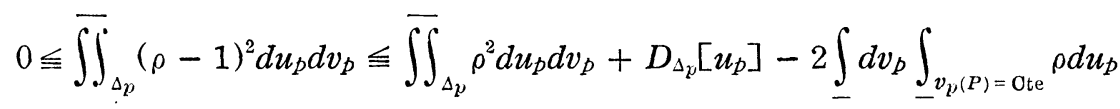

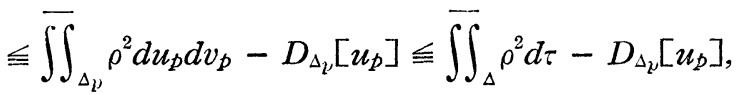

d'où $D_{\Delta_{p}}\left[u_{p}\right] \leqq M\{\gamma\}$; on a utilisé le fait que toutes les trajectoires orthogonales $v_{p}(P)=$ Cte, sauf au plus un nombre fini d'exceptions, des courbes de niveau de $u_{p}(P)$ sont des courbes joignant $c_{1}$ et $c_{2}$ dans $\Delta_{p}$. Lorsque $p \rightarrow \infty$, il en résulte que $D_{\Delta}\left[u_{0}\right] \leqq 1 / \lambda\{r\}$. La comparaison avec l'inégalité inverse conduit à l'égalité.

Le cas où $\Delta$ est compact dans $\widetilde{\mho}$ peut être traité très simplement.

Ainsi :

THÉORÈme 3. Soient $\widetilde{\mho}$ une surface de Riemann, $c_{1}$ et $c_{2}$ deux ensembles finis ou dénombrables de courbes régulières disjointes tels qu'ils forment la frontière, rélative à $\widetilde{\gamma}$, d'un ensemble ouvert $\Delta$ dans $\widetilde{\gamma}$, et $\mathfrak{l}$ l'ensemble des fontions $\{u(P)\}$ harmoniques dans $\Delta$ telles que $\varlimsup_{c_{1}} u(P) \leqq 0$ et $\frac{\lim }{c_{2}} u(P) \geqq 1$. Alors, quel que soit $u(P) \in \mathfrak{H}, v(P)$ etant la fonction conjuguee de $u(P)$, on a

$$
\int_{0}^{1} \frac{d u}{\int_{C(u)} d v} \leqq \frac{1}{\lambda\{c\}}=\lambda\{\gamma\},
$$

où $\{c\}$ est la famille des systèmes de courbes dans $\Delta$ homologues à $c_{1}$, à une partie de la frontière idéale de $\Delta$ près, et $\{\gamma\}$ est la famille des courbes qui joignent $c_{1}$ et $c_{2}$ dans $\Delta ; \lambda\{\gamma\}$ est la distance extrémale de $c_{1}$ et $c_{2}$ par rapport $\grave{a}$ 4. De plus, il existe une fonction extrémale $u_{0}(P) \in \mathfrak{U}$ qui donne la valeur 
maximum, égale à $\lambda\{\gamma\}$, à l'intégrale à gauche et dont l'intégrale de Dirichlet est égale à $\lambda\{c\}$.

5. On revient maintenant à la suite $\left\{D_{n}\right\}$ définie dans $\S 3$ sur une surface de Riemann $\tilde{F}$, et à l'élément $\mathfrak{Q}$ défini par $\left\{D_{n}\right\}$. Désignons par $\left\{c^{n}\right\}$ la famille des systèmes de courbes dans $D_{1}-D_{n}-c_{n}=\Delta_{n}$, homologues à $c_{1}$ à une partie de la frontière idéale de $\Delta_{n}$ près, par $\left\{\gamma^{n}\right\}$ la famille des courbes joignant $c_{1}$ et $c_{n}$ dans $\Delta_{n}$ et par $\{r\}$ la famille des courbes partant de $c_{1}$ et s'étendant vers 2. $^{8)} \quad$ En vertu des propriétés a) et d) de longueur extrémale, $\lambda_{\pi}\left\{c^{n}\right\}$ $€$ st décroissante mais reste supérieure à $\lambda_{\pi}\left(\bigcup_{n}\left\{c^{n}\right\}\right)$, et $\lambda_{\pi}\left\{r^{n}\right\}$ est croissante mais reste inférieure à $\lambda_{\pi}\{\gamma\}$, où $\pi$ est une fonction réelle non négative quelconque sur $D_{1}$. On pose ici les questions si $\lim _{n \rightarrow \infty} \lambda_{\pi}\left\{c^{n}\right\}=\lambda_{\pi}\left(\bigcup_{n}\left\{c^{n}\right\}\right)$ et si $\lim _{n \rightarrow \infty}$ $\lambda_{\pi}\left\{\gamma^{n}\right\}=\lambda_{\pi}\{\gamma\}$. On ne connait la réponse que dans le cas suivant très particulier.

ThÉoRème 4. Soient $\widetilde{F}$ une surface de Riemann, $\left\{D_{n}\right\}$ une suite décroissante d'ensembles ouverts de frontières relatives disjointes $\left\{c_{n}\right\},\left\{c^{n}\right\}$ la famille des systèmes de courbes dans $D_{1}-D_{n}-c_{n}=\Delta_{n}$ homologues à $c_{1}$, à une partie de la frontière idéale de $\Delta_{n}$ près, $\left\{\gamma^{n}\right\}$ la famille des courbes joignant $c_{1}$ et $c_{n}$ dans $\Delta_{n}$ et $\{r\}$ la famille des courbes partant de $c_{1}$ et s'étendant vers \& que $\left\{D_{n}\right\}$ détermine. Sil existe une surface de Riemann $\mathfrak{F}^{*} \supset \mathfrak{F}$ telle que tout $\Delta_{n}$ soit relativement compact par rapport $\grave{a} \mathfrak{F}^{*}$ et soit borné par un nombre fini de courbes régulières et si $\lambda\left\{c^{2}\right\}<\infty$, on a $\lim _{n \rightarrow \infty} \lambda\left\{c^{n}\right\}=\lim _{n \rightarrow \infty} 1 / \lambda\left\{r^{n}\right\}=\lambda\left(\bigcup_{n}\left\{c^{n}\right\}\right)=1 / \lambda\{r\}^{9}{ }^{9}$

On désigne par $\gamma_{n}$ la partie de la frontière de $\Delta_{n}$ dans $\mathfrak{F}^{*}$, différente de $c_{1}$ et de $c_{n}$. On prolonge $\Delta_{n}$ par symétrie le long de $\gamma_{n}$ et note $\hat{\Delta}_{n}$ le double ainsi obtenu, et $\hat{c}_{n}$ le double de $c_{n}$. $\hat{\Delta}_{n}$ consiste d'un nombre fini de surfaces de Riemann. ${ }^{10)}$ La condition $\lambda\left\{c^{n}\right\} \leqq \lambda\left\{c^{2}\right\}<\infty$ montre que, sur $\hat{\Delta}_{n}, \hat{c}_{1}$ et $\hat{c}_{n}$ sont disjointes. Par conséquent, l'intégrale de Dirichlet de la mesure harmonique $\hat{\omega}_{n}(P)$ de $\hat{c}_{n}$ sur $\hat{\Delta}_{n}$ est finie. La restriction dans $\Delta_{n}$ de $\hat{\omega}_{n}(P)$ est égale à la fonction $u^{(n)}(P)$ harmonique bornée dans $\Delta_{n}$, égale à 0 sur $c_{1}$ et à 1 sur $c_{n}$ et ayant la dérivée normale qui s'annule sur $\gamma_{n}$. Par suite on voit facilement que $(\infty>) D_{\Delta_{n}}\left[u^{(n)}\right] \leqq D_{\Delta_{n}}[u]$ pour toute fonction $u(P)$ de la famille $\mathfrak{u}_{n}$ des fonctions $\{u(P)\}$ harmoniques dans $\Delta_{n}$ telles que $\varlimsup_{c_{1}} u(P) \leqq 0$ et $\lim _{c_{2}} u(P) \geqq 1$. Parmi les fonctions de $\mathfrak{U}_{n}$, on a trouvé dans $\S 4$ la fonction unique qui possède l'intégrale de Dirichlet minimale, égale à $\lambda\left\{c^{n}\right\}<\infty$. Cette fonction est donc

8) Cela veut dire qu'il existe une suite de points sur $\gamma$ qui converge vers $\mathfrak{L}$.

9) On peut appeler $\lambda\{\gamma\}$ la distance extrémale de $c_{1}$ et $\mathbf{L}$.

10) Si la frontière d'une composante connexe $\delta$ de $\Delta_{n}$ ne contient aucune courbe composante de $\gamma_{n}$, on n'a pas de surface de Riemann qui est le double de $\delta$. La modification pour ce cas est facile; on emploiera des terminologies, méme dans ce cas, comme si $\delta$ possédait son double. 
identique à $u^{(n)}(P)$. On pose $\Delta=\bigcup_{n} \Delta_{n}$ et $\hat{\Delta}=\bigcup_{n} \hat{\Delta}_{n}$. Soit $\hat{\Gamma}$ la partie de la frontière de $\hat{\Delta}$, différente de $\hat{c}_{1}$, et soit $\hat{\omega}(P)$ la mesure harmonique de $\hat{\Gamma}$ sur $\hat{\Delta}$. La restriction de $\hat{\omega}(P)$ dans $\Delta$ est égale à $u^{0}(P)=\lim _{n \rightarrow \infty} u^{(n)}(P)$. Si $u^{0}(P) \equiv 0$ sur $\Delta, \lambda\{r\} \geqq \lim _{n \rightarrow \infty} \lambda\left\{\gamma^{n}\right\}=\infty$ et $\lambda\left(\bigcup_{n}\left\{c^{n}\right\}\right) \leqq \lim _{n \rightarrow \infty} \lambda\left\{c^{n}\right\}=0$. En supposant donc que $u^{0}(P) \neq 0$, nous désignons par $v^{0}(P)$ la fonction conjuguée de $u^{0}(P)$ sur la partie $\Delta^{\prime} \subset \Delta$ où $u^{0}(P)$ n'est pas constante. Or, il est connu (voir [2]) que sur le double $\Delta^{\prime}$ de $\Delta^{\prime}$ presque toute la trajectoire orthogonale des courbes de niveau $\hat{\omega}(P)$ $=$ Cte est une courbe partant de $c_{1}$ et convergeant vers $\hat{\Gamma}$ sur laquelle $\hat{\omega}(P) \rightarrow 1$. Comme $\hat{b}^{\prime}$ est symétrique, les trajectoires sont aussi symétriques par rapport à $\gamma_{0}=\bigcup_{n} \gamma_{n}$, et presque toutes les trajectoires orthogonales convergent vers $\mathbb{Z}$ sur $\Delta^{\prime}$.

On note que $M\{r\}<\infty$, puisque $\lambda\{r\} \geqq \lambda\left\{\gamma^{2}\right\}>0$. Etant donné $\varepsilon>0$, soit $\rho$ une répartition en concurrence pour $\{\gamma\}$ sur $\Delta$ telle que $\varepsilon+M\langle\gamma\} \geqq \iint \rho^{2} d u^{0} d v^{0}$. Par l'inégalité de Schwarz, on a pour presque toute valeur de $v^{0}, 0<v^{0}<\int_{c_{1}} d v^{0}$,

$$
1 \leqq\left(\int_{v^{0}=\mathrm{Cte}} \rho d u^{0}\right)^{2} \leqq \overline{\int_{0}} \rho^{2} d u^{0} \int_{0}^{1} d u^{0}
$$

et donc $\int_{c_{1}} d v^{0} \leqq \iint_{\int} \rho^{2} d u^{0} d v^{0} \leqq M\{\gamma\}+\varepsilon$. D'après le théorème $3 \lambda\left\{c^{n}\right\}=\int_{c_{1}} d v^{(n)}$ et ceci tend vers $\int_{c_{1}} d v^{0}$. $\varepsilon$ étant arbitrairement petit, on a $\int_{c_{1}} d v^{0}=\lim _{n \rightarrow \infty} \lambda\left\{c^{n}\right\}$ $=\lim _{n \rightarrow \infty} 1 / \lambda\left\{\gamma^{n}\right\} \leqq 1 / \lambda\{\gamma\}$. La comparaison avec l'inégalité inverse $\lambda\left\{\gamma^{n}\right\} \leqq \lambda\{\gamma\}$ nous donne que $\lim _{n \rightarrow \infty} \lambda\left\{c^{n}\right\}=\lim _{n \rightarrow \infty} 1 / \lambda\left\{r^{n}\right\}=1 / \lambda\{r\}$. Comme $\rho=1 / \int_{c_{1}} d v^{0}$ est une répartition en concurrence pour $\bigcup_{n}\left\{c^{n}\right\}$ par rapport à $u^{0}+i v^{0}, 1 / \lambda\left(\bigcup_{n}\left\{c^{n}\right\}\right)$ $\leqq \iint \rho^{2} d u^{0} d v^{0}=1 / \int_{c_{1}} d v^{0}=\lambda\{\gamma\}$. L'inégalité inverse $1 / \lambda\{\gamma\}=\lim _{u \rightarrow \infty} \lambda\left\{c^{n}\right\} \geqq \lambda\left(\bigcup_{n}\left\{c^{n}\right\}\right)$ est déjà connue et il en résulte toutes les égalités. Ainsi le théorème est démontré.

Corollaire 1. Soit $D$ un domaine sur une surface de Riemann $\mathfrak{F}$, borné par un nombre fini ou dénombrable de courbes régulières non s'accumulant sur ঋ. Alors la surface symétrique $\hat{D}$ est parabolique si et seulement si la distance extrémale, par rapport à $D$, d'un ensemble compact, borné par une courbe dans $D$, et la frontière idéale de $D$ est infinie.

En particulier,

Corollaire 2. Une surface de Riemann $\tilde{\mho}$ est parabolique si et seulement si la distance extrémale d'un ensemble compact borné par une courbe dans $\widetilde{\mho}$ et 
la frontière idéale de $\tilde{\wp}$ est infinie. ${ }^{11}$

6. On va étudier plus profondément des représentations paraboliques $\left(\mathfrak{F}_{\mathfrak{R}}\right)$ définies dans [A].

D'abord il s'agit de la définition générale de transformations quasi-conformes, due à Pfuger [6] et Ahlfors [1]. ${ }^{12)}$ Soit $T(z)$ un homéomorphisme d'un domaine $D$ du plan $z=x+i y$ sur un domaine $G$ du plan $w=u+i v$. On appelle un domaine de Jordan avec quatre points $z_{1}, z_{2}, z_{3}$ et $z_{4}$ différents marqués sur la frontière un quadrilatère et chacune de quatre parties entre $z_{1}, z_{2}, z_{3}$ et $z_{1}$ un côté. Le module Mod $(Q)$ d'un quadrilatère $Q=\left(z_{1}, z_{2}, z_{3}, z_{4}\right)$ est défini par la distance extrémale de deux côtés $z_{1} z_{2}$ et $z_{3} z_{4}$. On suppose que, quel que soit un domaine $D^{\prime}$ relativement compact par rapport à $D, \sup _{Q}[\operatorname{Mod}(Q) / \operatorname{Mod}$ $(T(Q))]=K\left(D^{\prime}\right)<+\infty$ pourtout qu adrilatère $Q$ dans $D^{\prime}$. On pose $K(z)=$ $\inf _{z \in D^{\prime}} K\left(D^{\prime}\right)$. Il est facile de généraliser la définition au cas où un homéomorphisme d'une surface de Riemann sur une autre est donné. Pour $T(z)=u(z)$ $+i v(z)$ défini ci-dessus dans $D$, Mori [4] a démontré que

i) $T(z)$ est totalement dérivable presque partout dans $D$;

ii) en chaque point $z$, où $T(z)$ est totalement dérivable, on a

$$
\operatorname{Max}_{0 \leqq \theta \leqq 2 \pi}\left|D_{\partial} w\right|^{2} \leqq K(z)|J(z)| ;
$$

iii) pour presque toute valeur $y=y_{0}, T\left(x, y_{0}\right)$ est une fonction abosolument continue de $x$ sur l'intersection de la ligne $y=y_{0}$ et de $D$.

Maintenant on s'occupe du cas où l'application est non nécessairement univalente, comme dans $\S 1$ de $[\mathrm{A}]$. Soit $f(\widetilde{P})$ une application continue, dans une surface de Riemann $\mathfrak{R}^{13)}$ d'un nombre fini ou dénombrable de surfaces de Riemann $\widetilde{\mathscr{V}}$. Soit $\widetilde{E}$ un ensemble fermé don t'image $\underline{E}$ par $f(\widetilde{P})$ est de mesure linéaire nulle dans $\mathfrak{R}$. Supposons que la restriction de $f(\widetilde{P})$ à $\widetilde{\widetilde{F}}-\widetilde{E}$ soit une transformation intérieure au sens de Stoïlow. On définira un espace topologique $\widetilde{\mho}^{*}$ comme suivant. On fait correspondre $(\widetilde{P}, f(\widetilde{P}))$ à $\widetilde{P} \in \widetilde{\mathfrak{l}}-\widetilde{E}$ et $\left(\widetilde{E}_{\alpha}, f\left(\widetilde{E}_{\alpha}\right)\right)$ à une composante connexe $\widetilde{E}_{\alpha}$ de $\widetilde{E}$; nous considérons ces couples comme les points de $\widetilde{F}^{*}$; comme $f(\widetilde{P})$ est continue et $f(\widetilde{E})=\underline{E}$ est totalement discontinue dans $\mathfrak{R}, f\left(\widetilde{E}_{\alpha}\right)$ est toujours un point. On définit un voisinage $V(\widetilde{V})$ de $\left(\widetilde{P}_{0}, f\left(\widetilde{P}_{0}\right)\right)$ par $\left\{(\widetilde{P}, f(\widetilde{P})) ; \widetilde{P} \in\right.$ un voisinage $\widetilde{V}$ de $\left.\widetilde{P}_{0}\right\}$ et un voisinage $V\left(\widetilde{V}^{\prime}\right)$ de $\left(\widetilde{E}_{\alpha}, f\left(\widetilde{E}_{\alpha}\right)\right)$ par $\left\{(\widetilde{P}, f(\widetilde{P}))\right.$ ou $\left(\widetilde{E}_{\beta}, f\left(\widetilde{E}_{\beta}\right)\right) ; \widetilde{P}$ ou $\widetilde{E}_{\beta}$ est compris dans un ensemble ouvert $\widetilde{V}^{\prime} \supset \widetilde{E}_{\alpha}$, dont la frontière est disjointe de toute composante de $\widetilde{E}$ \}. Les voisinages $V(\widetilde{V})$ et $V\left(\widetilde{V}^{\prime}\right)$ engendrent les ensembles des voisinages

11) Voir [7] à ce propos.

12) Pour la définition générale de transformations quasi-conformes, voir aussi [4], [10], [11].

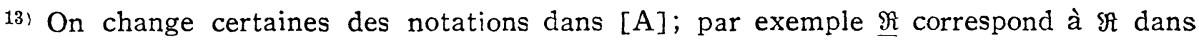
[A], $E$ à $E, \widetilde{\Im}$ à $\widetilde{G}$, etc. 
des points de $\mathfrak{F}^{*}$ et donc une topologie est définie sur $\mathfrak{F}^{*}{ }^{14}$ En définissant des paramètres locaux sur la partie $\widetilde{f}$ de $\mathfrak{F}^{*}$ correspondant à $\widetilde{F}-\widetilde{E}$ à la manière usuelle, $\widetilde{F}$ devient un nombre fini ou dénombrable de surfaces de Riemann

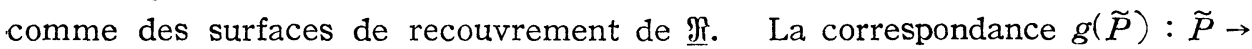
$(\widetilde{P}, f(\widetilde{P}))$ lorsque $\widetilde{P} \in \widetilde{\widetilde{J}}-\widetilde{E}$, et $\widetilde{P} \rightarrow\left(\widetilde{E}_{\alpha}, f\left(\widetilde{E}_{\alpha}\right)\right)$ lorsque $\widetilde{P} \in \widetilde{E}_{\alpha}$, est une application continue de $\widetilde{\mathfrak{F}}$ sur $\widetilde{F}^{*}$, et la correspondance $H\left(P^{*}\right): P^{*}=(\widetilde{P}, f(\widetilde{P})) \rightarrow f(\widetilde{P})$ ou $P^{*}=\left(\widetilde{E}_{\alpha}, f\left(\widetilde{E}_{\alpha}\right)\right) \rightarrow f\left(\widetilde{E}_{\alpha}\right)$ est aussi une application continue de $\widetilde{F}^{*}$ dans $\underline{\Re}$.

Supposons que la restriction de $f(\widetilde{P})$ à $\widetilde{\mho}-\widetilde{E}$ soit une fonction localement pseudo-analytique au sens de Pfluger-Ahlfors; c'est-à-dire que la restriction de $g(\widetilde{P})$ à $\widetilde{\mathfrak{F}}-\widetilde{E}$ satisfasse à la condition que nous venons de poser sur $T(z)$.

Soit $\left\{\underline{D}_{n}\right\}$ une suite décroissante d'ensembles ouverts dans $\underline{\Re}$ de frontières relatives $\left\{\underline{c}_{n}\right\}$ telles que chaque $\underline{s}_{n}$ se compose d'un nombre fini ou dénombrable de courbes régulières et $\underline{c}_{1} \cap \underline{c}_{n}=\phi$ pour tout $n \geqq 2$, et soit $\mathbb{Q}$ l'élément déterminé $\operatorname{par}\left\{\underline{D}_{n}\right\}$. On suppose que les images réciproques $\left\{c_{n}\right\}$ de $\left\{\underline{c}_{n}\right\}$ soient situées dans $\widetilde{\gamma}$; c'est-à-dire que $\left\{\underline{c}_{n}\right\}$ et $\underline{E}$ soient disjoints. Soit $\underline{u}_{n}(\underline{P})$ une fonction harmonique dans $\underline{D}_{1}-\underline{D}_{n}-\underline{c}_{n}$ telle que $\varlimsup_{\underline{c}_{1}} \underline{u}_{n}(\underline{P}) \leqq 0$ et $\frac{\lim }{c_{n}} \underline{u}_{n}(\underline{P}) \gtrsim 1$, et $c\left(\underline{u}_{n}\right)$ la courbe de niveau $\underline{u}_{n}(\underline{P})=$ Cte $\underline{u}_{n}, 0<\underline{u}_{n}<1$. On pose $u_{n}\left(P^{*}\right)=$ $\underline{u}_{n}\left(H\left(P^{*}\right)\right)$ et $c\left(u_{n}\right)=H^{-1}\left(c\left(\underline{u}_{n}\right)\right)$ et on désigne par $C_{n}$ la famille des $c\left(u_{n}\right)$ situées dans $\widetilde{\wp} ; c\left(\underline{u}_{n}\right)$ est disjointe de $\underline{E}$ pour presque toute valeur de $\underline{u}_{n}$, puisque $\underline{E}$ est de mesure linéaire nulle. Soit $C_{n}^{\prime}$ la sous-famille de $C_{n}$ dont sur chaque élément $c\left(u_{n}\right)$, la restriction de $g^{-1}(P)$ à $\tilde{f}$ est totalement dérivable et absolument continue; par les propriétés i) et iii), $c\left(u_{n}\right) \in C_{n}^{\prime}$ pour presque toute valeur de $u_{n}$. Si $q(P)$ désigne le quotient de dilatation de $g^{-1}(P)$ défini dans $\tilde{\jmath}$ comme dans e) de $\S 2$, la condition

$$
\int_{0}^{1} \frac{d u_{n}}{\int_{C\left(u_{n}\right) \in C_{n}} q d v_{n}} \rightarrow \infty \quad \text { avec } n,
$$

entraìne le fait: $\lambda_{1 / q}\left(C_{n}^{\prime}\right) \rightarrow 0$ d'après le théorème 2 , où $v_{n}(P)$ est la fonction conjuguée de $u_{n}(P)$ définie sur $\widetilde{F}$.

Or, on appellera une représentation continue $f(\widetilde{P})$ de $\widetilde{\mho}$ dans $\mathfrak{R}$, parabolique ( $\Omega$ ) si elle est localement pseudo-analytique au sens de Pfuger-Ahifors en dehors d'un ensemble $\widetilde{E}$ fermé, ayant l'image $\underline{E}$ de mesure linéaire nulle, et s'il existe une suite $\left\{\underline{D}_{n}\right\}$ déterminant $\underline{\mathfrak{Q}}^{15)}$ telle que $f(\widetilde{\widetilde{W}}) \cap \underline{c}_{1} \neq \phi$ et $\left\{\underline{u}_{n}(\underline{P})\right\}$, harmoniques

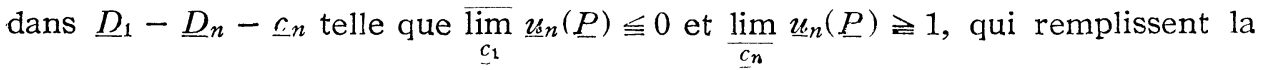
condition suivante, visiblement équivalente à (4):

$$
\int_{0}^{1} \frac{d \underline{v}_{n}}{\int \widetilde{q} d \underline{v}_{n}} \rightarrow \infty \quad \text { avec } n,
$$

14) On peut prouver que cette topologie est séparée.

15) On dit que deux suites déterminent le même $\underline{\Omega}$ si elles engendrent le même filtre. 
où $\tilde{q}$ est le quotient de dilatation de $f(\widetilde{P})$ réduite à $\widetilde{\mho}-\widetilde{E}$ et, $\underline{v}_{n}(\underline{P})$ étant la fonction conjuguée de $\underline{u}_{n}(\underline{P})$, l'intégrale $\int \widetilde{q} d \underline{v}_{n}=\int \underline{\widetilde{q}}(\widetilde{P}) d \underline{v}_{n}(f(\widetilde{P}))$ est prise sur l'image réciproque de $c\left(\underline{v}_{n}\right)$, qui est un système de courbes pour presque toute valeur de $\underline{u}_{n}, 0<\underline{u}_{n}<1$; on note que $\widetilde{a}(\widetilde{P})$ est égal à $q(P)$ en points correspondants. Une représentation parabolique $\left(\mathfrak{F}_{\mathfrak{R}}\right)$ dans $[\mathrm{A}]$ peut s'interpréter comme une telle représentation.

Ainsi on est arrivé à la position de pouvoir généraliser le théorème 1 de [A].

THÉORÈme 5. Soit $\mathfrak{\Re}$ une surface de Riemann et soit $f(z)$ une représentation parabolique $(\Omega)$ dans $\underline{\Re}$ d'un domaine $G$ du plan $z$, de la forme: $0<x<1,0$ $<y<h(x) \leqq \infty$. Si pour chaque valeur $x$ d'un sous-ensemble $X$ de l'intervalle $0<x<1$, on a $h(x)<\infty$ et s'il existe une suite $y_{p} \rightarrow h(x)$ telle que $f\left(x+i y_{p}\right)$ tende vers $\cong$ lorsque $p \rightarrow \infty$ pour tout $x \in X$, alors $m(X)=0$.

En effet, si $f\left(x+i y_{p}\right) \rightarrow \mathfrak{Q}$ l'image $g\left(x+i y_{p}\right)$ sur $\mathfrak{F}^{*}$, définie ci-dessus, tend vers $\mathfrak{L}$ que $\left\{D_{n}\right\}$, où $D_{n}=H^{-1}\left(\underline{D}_{n}\right)$, détermine. Nous avons vu que (4), équivalente à $(5)$, entraîne $\lambda_{1 / q}\left(C_{n}\right) \rightarrow 0$, d'où on conclut $m(X)=0$ par le théorème 1 .

7. Une fois la généralisation du théorème 1 de [A] étant obtenue, les théorèmes $2,3,4$ et 7 de [A] peuvent être généralisés pour des représentations paraboliques $(\underline{\Omega})$; ici on ne les énonce pas. Cependant, on s'intéresse à la généralisation du théorème 5 de $[\mathrm{A}]$.

Dans le cas particulier où $\mathscr{\&}$ peut être identifié à un point sur une surface de Riemann, la condition (5) est locale et écrite au moyen d'un paramètre local: soit en $\omega$. On pose $\underline{D}_{n}=\{|\omega|<1 / n\}$ et $\underline{c}_{n}=\{|\omega|=1 / n\}$; l'intégrale dans (5) s'écrit $\int_{1 ! n}^{1} \frac{d t}{q t d \varphi}\left(\omega=t e^{i / \text { }}\right)$, où l'intégrale du dénominateur est égale à la longueur totale de densité $q$ des courbes sur $\tilde{F}$ situées au-dessus de $|\omega|=t$, et elle sera notée $\lambda(t){ }^{16)} \quad$ L'intégrale $\int_{0}^{t} \int t d \varphi d t=\int_{0}^{t} \lambda(t) d t$ est égale à l'aire de densité $q$ de la partie de $\mathfrak{F}$, qui se projette dans $|\omega|<t$; on la désignera par $S(t)$.

Soit $1>t_{1}>t_{1}^{\prime} \geqq t_{2}>t_{2}^{\prime} \geqslant t_{3} \ldots \rightarrow 0$. Par l'inégalité de Schwarz on a

$$
\left(t_{\nu}-t_{\nu}^{\prime}\right)^{2}=\left(\int_{t_{\nu}^{\prime}}^{t_{\nu}} d t\right)^{2} \leqq \int_{t_{\nu}}^{t_{\nu}} \lambda d t \int_{t_{\nu}^{\prime}}^{t_{\nu}} \frac{d t}{\lambda}=\left\{S\left(t_{\nu}\right)-S\left(t_{\nu}^{\prime}\right)\right\} \int_{t_{\nu}^{\prime}}^{t_{\nu}} \frac{d t}{\lambda} .
$$

Donc

$$
\sum_{\nu=1}^{\infty} \frac{\left(t_{\nu}-t_{\nu}^{\prime}\right)^{2}}{S\left(t_{\nu}\right)-S\left(t_{\nu}^{\prime}\right)} \leqq \int_{0}^{1} \frac{d t}{\lambda}
$$

16) Cela n'est pas la longueur extrémale. 
$\mathrm{Si}$ on peut choisir $t_{\nu}$ et $t_{\nu}^{\prime}$ tels que la série à gauche diverge, la condition (5) sera satisfaite. Il en est ainsi lorsque $t_{\nu}^{\prime} / t_{\nu}<\kappa<1$ et $\sum_{\nu=1}^{\infty} t_{\nu}^{2} / S\left(t_{\nu}\right)=\infty$. Ceci est vrai si $\lim _{t \rightarrow 0} S(t) / t^{2}<\infty$ comme dans le théorème 5 de [A]; en effet, prenant $t_{1}>t_{1}^{\prime}=\frac{t_{1}}{2} \geqq t_{2}>t_{2}^{\prime}=\frac{t_{2}}{2} \gtrsim \ldots$ tels que $S\left(t_{\nu}\right) / t_{\nu}^{2}<K<\infty$, on a $\sum_{\nu=1}^{\infty} t_{\nu}^{2} / S(t)>$ $\sum_{\nu=1}^{\infty} 1 / K=\infty$. D'autre part Pfluger [7] a démontré récemment que si $\omega=f(\zeta)$ est une fonction non constante méromorphe dans $|\zeta|<1$, si l'aire sphèrique de son image est finie et si $S(t)=o\left(t^{2} \log 1 / t\right)$, alors l'ensemble des points sur $|\zeta|=1$ où $\lim _{r \rightarrow 1} f\left(r e^{i \theta}\right)=0\left(\zeta=r e^{i \theta}\right)$ est de capacité logarithmique (extérieure) nulle. Le théorème 6 montrera que l'aire sphèrique n'en faut pas être finie et qu'on peut améliorer sa condition sur $S(t)$ en la remplaçant par $S(t)=\mathrm{O}^{\prime}\left(t^{2} \log 1 / t\right)$. On note que, si on pose $t_{\nu}=t_{\nu-1}^{\prime}=e^{-\nu}$, on a $t_{\nu}^{\prime} / t_{\nu}=e^{-1}$ et $\sum_{\nu=1}^{\infty} e^{-2 \nu} / S\left(e^{-\nu}\right) \geqslant \frac{1}{M} \sum_{\nu=1}^{\infty} \frac{1}{\nu}$ $=\infty$, où $M$ est une constante finie telle que $S(t) \leqq M t^{2} \log 1 / t$. On peut encore relâcher la condition: $S\left(e^{-\nu}\right)=0\left(e^{-\nu} h(\nu)\right)$ avec $h(\nu)>0$ tel que $\sum_{\nu=1}^{\infty} 1 / h(\nu)=\infty$. Cependant, aucune de cette condition et la condition $\lim _{t \rightarrow 0} S(t) / t^{2}<\infty$ n'entraîne l'autre.

On énonce une généralisation du théorème 5 de $[\mathrm{A}]$ comme suivant:

THÉORÈME 6. Soit $f(\zeta)$ une fonction non constante continue definie dans

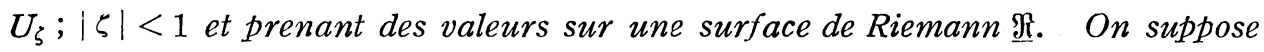
de plus que $f(\zeta)$ soit une représentation localement pseudo-analytique au sens de Pfluger-Ahlfors en dehors d'un ensemble $E_{\zeta}$ fermé dans $U_{\zeta}$ ayant l'image $\underline{E}$ dans $\mathfrak{R}$ de mesure linéaire nulle. On désigne par $S(t)$ l'aire euclidienne de densité q, égale au quotient de dilatation, de la partie des surfaces de Riemann homéomorphes à $U_{\zeta}-E_{\zeta}$, qui se projette dans le cercle $|\omega|<t<1$, où $\omega$ est un paramètre local fixé tel que $\omega=0$ corresponde à $\underline{P} \in \underline{\Re}$. Si on peut choisir $1>t_{1}>t_{1}^{\prime} \geq t_{2}>t_{2}^{\prime} \gtrsim t_{3} \ldots \rightarrow 0$, tels que la série à gauche dans $(6)$ diverge, l'ensemble des valeurs sur $|\zeta|=1$ où $\lim _{r \rightarrow 1} f\left(r e^{i 0}\right)=\underline{P}\left(\zeta=r e^{i 0}\right)$ est de capacité logarithmique (extérieure) nulle.

La même idée que dans la démonstration du théorème 5 de [A] s'appliquera si le lemme suivant est établit; on n'a pas besoin de supprimer $\underline{P}$ de $\underline{R}$, ce que nous avons fait dans $[\mathrm{A}]$, puisque $\&$ peut être identifié à un point intérieur de $\underline{\Re}$ dans le présent mémoire.

Lemme 4. Sous les conditions dans le théorème 6 , si $f(\zeta)$ a la limite $\underline{P}$ radialement à un point $e^{i \theta}$, l'image de toute courbe aboutissant $\grave{a} e^{i \theta}$ dans $U_{\zeta}$ s'étend vers $\underline{P}$. 
Soit $|\omega|<1$ un cercle paramétrique, où $\omega=0$ correspond à $\underline{P}$. Supposons qu'il existe une courbe $l$ dans $U_{\zeta}$ aboutissant à $e^{i \theta}$ et disjointe au rayon $\bar{O}^{i \theta}$ telle que son image $f(\zeta)$ se trouve hors $|\omega|<1$. Nous représentons $U_{\zeta}$ par $\log \left(1-\zeta e^{-i \theta}\right)$ sur un domaine dans le plan $w=u+i v$. L'image $B$ est placée entre les droites $v=-\frac{\pi}{2}$ et $v=\frac{\pi}{2}$, et le rayon $\overline{O e}^{i \theta}$ correspond à $0<u<\infty$. On divise $B$ en parties se trouvant dans les bandes $0<u<1,1<u<2, \ldots$ et désigne par $B_{p}$ la partie dans $p<u<p+1 ; B_{p}$ est comprise dans le rectangle $p<u<p+1,-\frac{\pi}{2}<v<\frac{\pi}{2}$. Si $p$ est suffisamment grand, l'image dans $\underline{\Re}$ du segment $p<u<p+1$ est située dans un voisinage $|\omega|<1 / n$ de $\underline{P}$. L'image $r(t)$ dans $B_{p}$ de $|\omega|=t$ existe pour presque toute valeur $t$ de $1 / n<t<1$, et la longueur de toute $\gamma(t)$ est $\geqq 1$, puisque $\gamma(t)$ se coupe tous les segments, joignant l'axe des $u$ et l'image $w(l)$ et parallèles à l'axe des $v$. Donc la longueur extrémale de $\{r(t)\} \geqslant 1 / \pi$. Mais comme on l'a vu, la condition (6) montre que la longueur extrémale de poids $1 / q$ de la famille des systèmes de courbes sur les surfaces de recouvrement, se projetant sur $|\omega|=t, 1 / n<t<1$, tend vers 0 lorsque $n \rightarrow \infty$. La propriété e) dans $\S 2$ conduit à une contradiction.

On peut étendre le théorème 6 de $[\mathrm{A}]$ à la même manière. L'idée de la démonstration du lemme 4 sera reprise pour généraliser un théorème de Lindelöf dans un mémoire sous préparation.

Enfin je signale que j'ai l'intention de prendre d'autres occasions pour examiner les résultats dans $\S \S 8-9$ de [A] et améliorer les résultats sur les ensembles d'accumulation obtenus dans [5].

Addenda: après avoir complété ce mémoire, le travail suivant a été publié, qui est étroitement lié à nos $\S \S 4-5$ :

K. Strebel: Die extremale Distanz zweier Enden einer Riemann'schen Fläche, Ann. Acad. Sci. Fenn., A.I., (1955), no. 179, 21 pp.

\section{BiBLIOGRAPHIE}

[1] L. Ahlfors: On quasi conformal mappings, Journ. D’Anal. Math., 3 (1953-54), pp. 1-58; Corrections, pp. 207-208.

[2] M. Brelot et G. Choquet: Espaces et lignes de Green, Ann. Inst. Fourier, 3 (1952), pp. 199-263.

[3] J. Hersch: Longueurs extrémales et théorie des fonctions, Comment. Math. Helv., 29 (1955), pp. 301-337.

[4] A. Mori: On quasi-conformality and pseudo-analyticity, à paraitre.

[5] M. Ohtsuka: Sur les ensembles d'accumulation relatifs à des transformations plus générales que les transformations quasi-conformes, Ann. Inst. Fourier, 5 (1955), pp. 29-37.

[6] A. Pfluger: Quelques théorèmes sur une classe de fonctions pseudo-analytiques, C. R. Acad. Sci. Paris, 231 (1950). pp. 1022-1023.

[ 7 ] A. Pfluger: Extremallängen und Kapazität, Comment. Math. Helv., 29 (1955), pp. 120131. 
[ 8 ] T. Radó: Length and area, New York (1948).

[9] S. Saks: Theory of the integral, Varsovie (1937).

[10] Z. Yûjôbô: On pseudo-regular functions, Comment. Math. Univ. St. Pauli, 1 (1953), pp. 67-80.

[11] Z. Yûjôbô: On absolutely continuous functions of two or more variables in the Tonelli sense and quasi-conformal mappings in the A. Mori sense, ibid.. 4 (1955).

Institut de Mathematiques

Universite de Nagoya 